\title{
On Sets of Zeroes of Clifford Algebra-Valued Polynomials
}

\author{
Yan Yang and Tao Qian \\ School of Mathematics and Computational Science, Sun Yat-Sen University, and \\ Department of Mathematics, Faculty of Science and Technology, University of Macau \\ E-mail: mathyy@sina.com, fsttq@umac.mo
}

\begin{abstract}
In this note we study zeroes of Clifford algebra-valued polynomials. We prove that if such a polynomial has only real coefficients, then it has two types of zeroes: the real isolated zeroes and the spherical conjugate ones. The total number of zeroes does not exceed the degree of the polynomial. We also present a technique for computing the zeroes.
\end{abstract}

keywords : Clifford algebra, zeroes of polynomials

AMS subject classification : $30 \mathrm{G} 35,32 \mathrm{~A} 05$

\section{Introduction}

There has been an ample amount of literature discussing zeros of polynomials. This note concerns about polynomials of one hyper-complex-variable (hyper-complex valued polynomials), while the coefficients may be real, or complex, or hyper-complex constants. By hyper-complex numbers we mean quaternions, octonions, several complex variables or Clifford numbers. Niven in [3, 4] first studied zeroes of hyper-complex polynomials which further led to the article by Eilenberg and Niven [5] where a fundamental theorem for quaternionic polynomials was established. In [6], they proved that any quaternionic polynomial of degree $n \geq 1$ has at least one zero and there should be two types of zeroes: They are either isolated or spherical ones. In [7] zeroes of quaternionic and octonionic polynomials with real coefficients are also studied. In that paper, the authors found that the root-set of such a polynomial is a union of a finite number of codimension 2 Euclidean spheres together with a finite number of real points. In [8], roots of polynomials with bicomplex coefficients are studied. To the authors knowledge, in the higher dimensional cases under the structure of Clifford algebra, there has been no such results.

In this article, we first study zeroes of Clifford algebra-valued polynomials. For the special cases, we consider paravector-valued polynomials with real coefficients. Using a technical method, we introduce a one-to-one correspondence between such a polynomial and a complex polynomial and then extend the results in [6].

\footnotetext{
${ }^{1}$ This project sponsored by the Scientific Research Foundation for the Youth Scholars of Sun Yat-Sen University and by Research Grant of University of Macau on Applications of Hyper-Complex Analysis (cativo: 7560 ).
} 
We first give some basic knowledge in relation to Clifford algebra $([1,2])$. Let $\mathbf{e}_{1}, \ldots, \mathbf{e}_{m}$ be basic elements satisfying $\mathbf{e}_{i} \mathbf{e}_{j}+\mathbf{e}_{j} \mathbf{e}_{i}=-2 \delta_{i j}$, where $\delta_{i j}=1$ if $i=j$; and $\delta_{i j}=0$ otherwise, $i, j=1,2, \cdots, m$. Let

$$
\mathbf{R}^{m}=\left\{\underline{x}=x_{1} \mathbf{e}_{1}+\cdots+x_{m} \mathbf{e}_{m}: x_{j} \in \mathbf{R}, j=1,2, \cdots, m\right\}
$$

be identical with the usual Euclidean space $\mathbf{R}^{m}$, and

$$
\mathbf{R}_{1}^{m}=\left\{x=x_{0} \mathbf{e}_{0}+\underline{x}: x_{0} \in \mathbf{R}, \underline{x} \in \mathbf{R}^{m}\right\} \text {, where } \mathbf{e}_{0}=1 \text {. }
$$

An element in $\mathbf{R}_{1}^{m}$ is called a paravector. For $x \in \mathbf{R}_{1}^{m}$, it consists of a scalar part and a vector part. We use the dotations

$$
x_{0}=\operatorname{Sc}(x), \underline{x}=\operatorname{Vec}(x) .
$$

The real (or complex) Clifford algebra generated by $\mathbf{e}_{1}, \mathbf{e}_{2}, \cdots, \mathbf{e}_{m}$, denoted by $\mathbf{R}^{(m)}$ (or $\mathbf{C}^{(m)}$ ), is the associative algebra generated by $\mathbf{e}_{1}, \mathbf{e}_{2}, \cdots, \mathbf{e}_{m}$ over the real (or complex) field $\mathbf{R}$ (or $\mathbf{C}$ ). A general element in $\mathbf{R}^{(m)}$ (or $\mathbf{C}^{(m)}$ ), therefore, is of the form $x=$ $\sum_{S} x_{S} \mathbf{e}_{S}$, where $\mathbf{e}_{S}=\mathbf{e}_{i_{1}} \mathbf{e}_{i_{2}} \cdots \mathbf{e}_{i_{l}}, x_{S} \in \mathbf{R}$ (or $\mathbf{C}$ ), and $S$ runs over all the ordered subsets of $\{1,2, \cdots, m\}$, namely

$$
S=\left\{1 \leq i_{1}<i_{2}<\cdots<i_{l} \leq m\right\}, \quad 1 \leq l \leq m
$$

We define the conjugation of $\mathbf{e}_{S}$ to be $\overline{\mathbf{e}}_{S}=\overline{\mathbf{e}}_{i_{l}} \cdots \overline{\mathbf{e}}_{i_{1}}, \overline{\mathbf{e}}_{j}=-\mathbf{e}_{j}$. This induces the Clifford conjugate $\bar{x}=x_{0}-\underline{x}$ of a paravector $x=x_{0}+\underline{x}$.

The product between $x$ and $y$ in $\mathbf{R}_{1}^{m}$, denoted by $x y$ is split into three parts: a scalar part, a vector part and a bivector part, that is

$$
x y=\left(x_{0} y_{0}+\underline{x} \cdot \underline{y}\right)+\left(x_{0} \underline{y}+y_{o} \underline{x}\right)+\underline{x} \wedge \underline{y},
$$

where

$$
\begin{gathered}
\underline{x} \cdot \underline{y}=-\sum_{i=1}^{m} x_{i} y_{i}, \\
\underline{x} \wedge \underline{y}=\sum_{i=1}^{m} \sum_{j=i+1}^{m}\left(x_{i} y_{j}-x_{j} y_{i}\right) \mathbf{e}_{i} \mathbf{e}_{j} .
\end{gathered}
$$

In particular,

$$
x x=x_{0}^{2}-\sum_{i=1}^{m} x_{i}^{2}+2 x_{0} \underline{x}=2 x_{0} x-|x|^{2},
$$

where

$$
|x|^{2}=x \bar{x}=\sum_{i=0}^{m} x_{i}{ }^{2} .
$$

In the following, the so-called Clifford-Heaviside functions

$$
P^{ \pm}(\underline{x})=\frac{1}{2}\left(1 \pm \mathbf{i} \frac{\underline{x}}{|\underline{x}|}\right), \mathbf{i} \text { is the imaginary unit in } \mathbf{C}
$$


will play an important role, which were first introduced by Sommen in [9] and McIntosh in [10]. Introducing spherical coordinates in $\mathbf{R}^{m}$, we have $\underline{x}=r \underline{\omega}, r=|\underline{x}| \in[0, \infty), \underline{\omega} \in$ $S^{m-1}$, where $S^{m-1}$ is the unit sphere in $\mathbf{R}^{m}$. Thus,

$$
P^{ \pm}(\underline{\omega})=\frac{1}{2}(1 \pm \mathbf{i} \underline{\omega})
$$

They are self adjoint mutually orthogonal primitive idempotents:

$$
P^{+}(\underline{\omega})+P^{-}(\underline{\omega})=1, P^{+}(\underline{\omega}) P^{-}(\underline{\omega})=P^{-}(\underline{\omega}) P^{+}(\underline{\omega})=0, \quad\left(P^{ \pm}(\underline{\omega})\right)^{2}=P^{ \pm}(\underline{\omega}) .
$$

Furthermore, we have

$$
P^{ \pm}(\underline{\omega}) \underline{\omega}=\underline{\omega} P^{ \pm}(\underline{\omega})=\mp \mathbf{i} P^{ \pm}(\underline{\omega})
$$

and thus

$$
P^{ \pm}(\underline{\omega}) x=x P^{ \pm}(\underline{\omega})=P^{ \pm}\left(x_{0} \mp \mathbf{i}|\underline{x}|\right) .
$$

\section{The set of zeroes of a Clifford algbra-valued poly- nomial}

In this section, we will consider the following polynomials of degree $n \geq 1$ with paravector variable $x \in \mathbf{R}_{1}^{m}$ and paravector coefficients,

$$
R_{n}(x)=a_{n} x^{n}+a_{n-1} x^{n-1}+\cdots+a_{1} x+a_{0},
$$

and

$$
L_{n}(x)=x^{n} a_{n}+x^{n-1} a_{n-1}+\cdots+x a_{1}+a_{0},
$$

where $\left\{a_{0}, a_{1}, \cdots, a_{n}\right\} \subseteq \mathbf{R}_{1}^{m}$ and $a_{n} \neq 0$.

Since

$$
\overline{R_{n}(\bar{x})}=x^{n} \overline{a_{n}}+x^{n-1} \overline{a_{n-1}}+\cdots+x \overline{a_{1}}+\overline{a_{0}},
$$

it is enough to consider only one of the two polynomials.

First, we show that every polynomial $R_{n}$ has a special representation based on which our study is carried out.

For $x=x_{0}+\underline{x} \in \mathbf{R}_{1}^{m}$, we have

$$
\begin{aligned}
x^{2} & =2 x_{0} x-|x|^{2} \\
& =2 \operatorname{Sc}(x) x-|x|^{2} .
\end{aligned}
$$

Then

$$
\begin{aligned}
x^{3}=x^{2} x & =\left(2 \operatorname{Sc}(x) x-|x|^{2}\right) x \\
& =2 \operatorname{Sc}(x) x^{2}-|x|^{2} x \\
& =\left[(2 \operatorname{Sc}(x))^{2}-|x|^{2}\right] x-2 \operatorname{Sc}(x)|x|^{2}
\end{aligned}
$$


Going like this, we obtain the bi-axial form formula:

$$
x^{n}=A_{n}(x) x+B_{n}(x),
$$

where $A_{n}$ and $B_{n}$ are real-valued functions of $x$ defined by the recurrent formulas:

$$
\begin{aligned}
& A_{n+1}(x)=2 \operatorname{Sc}(x) A_{n}(x)-|x|^{2} A_{n-1}(x) \\
& B_{n+1}(x)=-|x|^{2} A_{n}(x),
\end{aligned}
$$

where

$$
\begin{aligned}
& A_{1}(x)=1 \\
& A_{2}(x)=2 \operatorname{Sc}(x) \\
& B_{1}(x)=0 \\
& B_{2}(x)=-|x|^{2} .
\end{aligned}
$$

Therefore,

$$
\begin{aligned}
R_{n}(x) & =a_{n}\left[A_{n}(x) x+B_{n}(x)\right]+a_{n-1}\left[A_{n-1}(x) x+B_{n-1}(x)\right]+\cdots+a_{1} x+a_{0} \\
& =A(x) x+B(x),
\end{aligned}
$$

where

$$
A(x)=\sum a_{i} A_{i}(x), B(x)=\sum a_{i} B_{i}(x) .
$$

Note that, like the coefficients, $A(x)$ and $B(x)$ are paravector-valued.

Remark 2.1 As a matter of fact, given any $x \in \mathbf{R}_{1}^{m}, A_{i}(x)$ and $B_{i}(x)$ depend not on $x$ but on its scalar part $x_{0}$ and the modulus of its vector part $|\underline{x}|$.

Thus, we have

Lemma 2.1 If two paravectors $x=x_{0}+\underline{x}, y=y_{0}+y$ with $x_{0}=y_{0},|\underline{x}|=|y|$, then $A_{i}(x)=A_{i}(y), B_{i}(x)=B_{i}(y)$ and hence $A(x)=A(y), B(x)=B(y)$. In particular, $A(x)=A(\bar{x}), B(x)=B(\bar{x})$.

We are interested in the properties of the zeroes of both polynomials (2). Let us see the following examples:

Example 1 For $x^{2}+1=0$, the zeroes in $\mathbf{R}_{1}^{m}$ are all the vectors of modulus 1 in $\mathbf{R}^{m}$. Thus its solutions are all the points on the unit sphere.

Example 2 Equation $x^{2}+\mathbf{e}_{2}=0$ has two isolated zeroes in $\mathbf{R}_{1}^{m}$,

$$
x_{1,2}= \pm \frac{\sqrt{2}}{2}\left(1-\mathbf{e}_{2}\right)
$$

Example 3 Equation $x^{2}+\mathbf{e}_{3} x+\mathbf{e}_{2}=0$ has no zeroes in $\mathbf{R}_{1}^{m}$. 
Remark 2.2 In [6], we know that any quaternionic polynomials of degree $n \geq 1$ has at least one zero. While, in higher dimensional cases under the structure of Clifford algebra, when $n \geq 1$, none of the polynomials (2) and (3) have zeroes.

Next, we will consider structure of the set of zeroes of a polynomial of the form (2) when it has zeroes.

Definition 2.1 If $w_{0}=\alpha+\operatorname{Vec}\left(w_{0}\right)$ and $w_{1}=\alpha+\operatorname{Vec}\left(w_{1}\right)$ are two different paravectors with $\left|\operatorname{Vec}\left(w_{0}\right)\right|=\left|\operatorname{Vec}\left(w_{1}\right)\right|$, then they are said to be spherical conjugate to each other.

Proposition 2.1 Assume that $w_{0}=\alpha+\operatorname{Vec}\left(w_{0}\right)$ and $w_{1}=\alpha+\operatorname{Vec}\left(w_{1}\right)$ are spherical conjugate to each other, and they both are zeroes of the polynomial (2), then any paravector that is spherical conjugate to $w_{0}$ is also a zero of (2).

Proof For $w_{0}$ and $w_{1}$, we have

$$
\begin{aligned}
& R_{n}\left(w_{0}\right)=0=A\left(w_{0}\right) w_{0}+B\left(w_{0}\right) \\
& R_{n}\left(w_{1}\right)=0=A\left(w_{1}\right) w_{1}+B\left(w_{1}\right)
\end{aligned}
$$

and

$$
A\left(w_{0}\right)=A\left(w_{1}\right), B\left(w_{0}\right)=B\left(w_{1}\right) .
$$

Hence $A\left(w_{0}\right) w_{0}+B\left(w_{0}\right)=A\left(w_{0}\right) w_{1}+B\left(w_{0}\right)$, which implies that

$$
A\left(w_{0}\right)\left[\operatorname{Vec}\left(w_{0}\right)-\operatorname{Vec}\left(w_{1}\right)\right]=0 .
$$

Since $w_{0}$ and $w_{1}$ are two different zeroes, the above means that $A\left(w_{0}\right)=0$ and then, from the recurrent formula, $B\left(w_{0}\right)=0$.

For any $w=\alpha+\operatorname{Vec}(w)$ with $|\operatorname{Vec}(w)|=\left|\operatorname{Vec}\left(w_{0}\right)\right|$, using Lemma 2.1 we obtain

$$
A(w)=A\left(w_{0}\right), B(w)=B\left(w_{0}\right) .
$$

Therefore, $R_{n}(w)=A(w) w+B(w)=A\left(w_{0}\right) w+B\left(w_{0}\right)=0$. This completes the proof.

Corollary 2.1 Let $w_{0}$ be a zero of the polynomial (2) such that $\operatorname{Vec}\left(w_{0}\right) \neq 0$ and that $\overline{w_{0}}$ is also a zero of it, then any spherical conjugate paravector to $w_{0}$ is also a zero of it.

Definition 2.2 Given a polynomial $R_{n}(x)$, then any of its zeroes generating a family of zeroes that are spherical conjugate to each other is called a spherical zero. A zero that is not spherical is called an isolated zero.

Proposition 2.2 The number of the isolated non-scalar zeroes of the polynomial (2) is less or equal to $n$.

Proof Let $w_{1}, w_{2}, \cdots, w_{n+1}$ be different non-scalar isolated zeroes of $R_{n}$. Considering the polynomial

$$
L_{n}(x)=\overline{R_{n}(\bar{x})}=x^{n} \overline{a_{n}}+x^{n-1} \overline{a_{n-1}}+\cdots+x \overline{a_{1}}+\overline{a_{0}}=\sum_{k=0}^{n} x^{k} \overline{a_{k}},
$$


using the properties $(1)$ of $P^{+}(\underline{\omega})$, we have

$$
\begin{aligned}
P^{+}(\underline{\omega}) L_{n}(x) & =\sum_{k=0}^{n} P^{+}(\underline{\omega}) x^{k} \overline{a_{k}} \\
& =\sum_{k=0}^{n} P^{+}(\underline{\omega})\left(x_{0}-\mathbf{i}|\underline{x}|\right)^{k} \overline{a_{k}} \\
& =P^{+}(\underline{\omega}) L_{n}\left(x_{0}-\mathbf{i}|\underline{x}|\right) .
\end{aligned}
$$

Similarly,

$$
R_{n}(x) P^{+}(\underline{\omega})=R_{n}\left(x_{0}-\mathbf{i}|\underline{x}|\right) P^{+}(\underline{\omega}) .
$$

Hence

$$
\begin{aligned}
P^{+}(\underline{\omega}) L_{n}(x) R_{n}(x) P^{+}(\underline{\omega}) & =P^{+}(\underline{\omega}) L_{n}\left(x_{0}-\mathbf{i}|\underline{x}|\right) R_{n}\left(x_{0}-\mathbf{i}|\underline{x}|\right) P^{+}(\underline{\omega}) \\
& =P^{+}(\underline{\omega}) F_{2 n}\left(x_{0}-\mathbf{i}|\underline{x}|\right) P^{+}(\underline{\omega}),
\end{aligned}
$$

where

$$
\begin{aligned}
F_{2 n}\left(x_{0}-\mathbf{i}|\underline{x}|\right) & =L_{n}\left(x_{0}-\mathbf{i}|\underline{x}|\right) R_{n}\left(x_{0}-\mathbf{i}|\underline{x}|\right) \\
& =\left[\sum_{k=0}^{n}\left(x_{0}-\mathbf{i}|\underline{x}|\right)^{k} \overline{a_{k}}\right]\left[\sum_{k=0}^{n} a_{k}\left(x_{0}-\mathbf{i}|\underline{x}|\right)^{k}\right] \\
& =\overline{a_{0}} a_{0}+\left(\overline{a_{0}} a_{1}+\overline{a_{1}} a_{0}\right)\left(x_{0}-\mathbf{i}|\underline{x}|\right)+\cdots+\overline{a_{n}} a_{n}\left(x_{0}-\mathbf{i}|\underline{x}|\right)^{2 n} \\
& =\sum_{k=0}^{2 n}\left(\sum_{i=0}^{k} \overline{a_{i}} a_{k-i}\right)\left(x_{0}-\mathbf{i}|\underline{x}|\right)^{2 k}
\end{aligned}
$$

is a polynomial of $x_{0}-\mathbf{i}|\underline{x}|$ of order $2 n$ with real coefficients.

Similar computation gives

$$
P^{-}(\underline{\omega}) L_{n}(x) R_{n}(x) P^{-}(\underline{\omega})=P^{-}(\underline{\omega}) F_{2 n}\left(x_{0}+\mathbf{i}|\underline{x}|\right) P^{-}(\underline{\omega}) .
$$

If $R_{n}\left(w_{i}\right)=0(i=1,2, \cdots, n+1)$, then

$$
\begin{aligned}
0 & =P^{+}\left(\underline{w_{i}}\right) L_{n}\left(w_{i}\right) R_{n}\left(w_{i}\right) P^{+}\left(\underline{w_{i}}\right) \\
& =P^{+}\left(\underline{w_{i}}\right) F_{2 n}\left[\operatorname{Sc}\left(w_{i}\right)-\mathbf{i}\left|\operatorname{Vec}\left(w_{i}\right)\right|\right] P^{+}\left(\underline{w_{i}}\right) \\
& =F_{2 n}\left[\operatorname{Sc}\left(w_{i}\right)-\mathbf{i}\left|\operatorname{Vec}\left(w_{i}\right)\right|\right]\left(P^{+}\left(\underline{w_{i}}\right)\right)^{2} \\
& =F_{2 n}\left[\operatorname{Sc}\left(w_{i}\right)-\mathbf{i}\left|\operatorname{Vec}\left(w_{i}\right)\right|\right] P^{+}\left(\underline{w_{i}}\right) .
\end{aligned}
$$

Since $F_{2 n}$ is a complex-valued polynomial and $P^{+}\left(\underline{w_{i}}\right) \neq 0$, we have

$$
F_{2 n}\left[\operatorname{Sc}\left(w_{i}\right)-\mathbf{i}\left|\operatorname{Vec}\left(w_{i}\right)\right|\right]=0
$$

Likewise, $F_{2 n}\left[\operatorname{Sc}\left(w_{i}\right)+\mathbf{i}\left|\operatorname{Vec}\left(w_{i}\right)\right|\right]=0$. Therefore, $F_{2 n}(z)$ has $2 n+2$ zeroes, which is a contradiction. This completes the proof.

We further have 
Proposition 2.3 The number of the isolated zeroes of the polynomial (2) is less or equal to $n$.

Proof Let $w_{1}, w_{2}, \cdots, w_{p}(1 \leq p \leq n+1)$ be different scalar zeroes and $w_{p+1}, \cdots, w_{n+1}$ be different non-scalar isolated zeroes of $R_{n}$. Then the polynomial can be written as

$$
R_{n}(x)=R_{n-p}(x)\left(x-w_{1}\right) \cdots\left(x-w_{p}\right) .
$$

If $R_{n}\left(w_{i}\right)=0, p+1 \leq i \leq n+1$, note that $w_{i}-w_{j}$ have inverse paravectors, where $p+1 \leq i \leq n+1,1 \leq j \leq p$, then $R_{n-p}\left(w_{i}\right)=0$. That means equation $R_{n-p}(x)=0$ has $n-p+1$ non-scalar isolated zeroes, which is a contradiction to Proposition 2.2. This completes the proof.

Theorem 2.1 The number of the isolated zeroes together with the double number of the spherical zeroes of polynomial (2) does not exceed the degree of the polynomial.

We will prove it in next section.

Next section, as a special cases, we will study sets of zeroes of polynomials with real coefficients.

\section{Sets of zeroes of polynomials with real coefficients}

Consider the polynomial

$$
Q_{n}(x)=a_{n} x^{n}+a_{n-1} x^{n-1}+\cdots+a_{1} x+a_{0},
$$

with $a_{i} \in \mathbf{R}$.

Using Corollary 2.1, we can obtain the following conclusion:

Proposition 3.1 Any zero of (4) with the form $w_{0}=\alpha+\operatorname{Vec}\left(w_{0}\right)$, $\operatorname{Vec}\left(w_{0}\right) \neq 0$, is a spherical zero.

Proof According to Corollary 2.1, it will suffice to show that $\overline{w_{0}}$ is also a zero of (4).

In fact, based on the previous discussion, $Q_{n}(x)$ has the representation

$$
Q_{n}(x)=A(x) x+B(x)
$$

where, based on the real-coefficients assumption, $A(x)$ and $B(x)$ are real numbers.

If $Q_{n}\left(w_{0}\right)=0$, then

$$
A\left(w_{0}\right) \alpha+B\left(w_{0}\right)=0, A\left(w_{0}\right) \operatorname{Vec}\left(w_{0}\right)=0,
$$

which implies $A\left(w_{0}\right)=B\left(w_{0}\right)=0$. 
From Lemma 2.1, we have

$$
A\left(w_{0}\right)=A\left(\overline{w_{0}}\right), B\left(w_{0}\right)=B\left(\overline{w_{0}}\right) .
$$

Therefore, $Q_{n}\left(\overline{w_{0}}\right)=A\left(\overline{w_{0}}\right) \overline{w_{0}}+B\left(\overline{w_{0}}\right)=0$.

From Proposition 3.1, we obtain

Corollary $3.1 Q_{n}(x)$ has no isolated non-scalar zeroes.

Next, we will introduce a method to solve the equation $Q_{n}(x)=0$.

For $Q_{n}(x)=\sum_{k=0}^{n} a_{k}\left(x_{0}+\underline{x}\right)^{k}, a_{k} \in \mathbf{R}$, we have

$$
\begin{aligned}
& P^{+}(\underline{\omega}) Q_{n}(x)=Q_{n}(x) P^{+}(\underline{\omega})=Q_{n}\left(x_{0}-\mathbf{i}|\underline{x}|\right) P^{+}(\underline{\omega}) \\
& P^{-}(\underline{\omega}) Q_{n}(x)=Q_{n}(x) P^{-}(\underline{\omega})=Q_{n}\left(x_{0}+\mathbf{i}|\underline{x}|\right) P^{-}(\underline{\omega}) .
\end{aligned}
$$

Using the properties of $P^{ \pm}(\underline{\omega})$, we have

$$
Q_{n}(x)=Q_{n}(x)\left[P^{+}(\underline{\omega})+P^{-}(\underline{\omega})\right]=Q_{n}\left(x_{0}-\mathbf{i}|\underline{x}|\right) P^{+}(\underline{\omega})+Q_{n}\left(x_{0}+\mathbf{i}|\underline{x}|\right) P^{-}(\underline{\omega}) .
$$

Thus

$$
\begin{aligned}
Q_{n}(x)=0 & \Longleftrightarrow Q_{n}\left(x_{0}-\mathbf{i}|\underline{x}|\right) P^{+}(\underline{\omega})+Q_{n}\left(x_{0}+\mathbf{i}|\underline{x}|\right) P^{-}(\underline{\omega})=0 \\
& \Longleftrightarrow Q_{n}\left(x_{0}-\mathbf{i}|\underline{x}|\right) P^{+}(\underline{\omega})=0 \text { and } Q_{n}\left(x_{0}+\mathbf{i}|\underline{x}|\right) P^{-}(\underline{\omega})=0 \\
& \Longleftrightarrow Q_{n}\left(x_{0}-\mathbf{i}|\underline{x}|\right)=0 \text { and } Q_{n}\left(x_{0}+\mathbf{i}|\underline{x}|\right)=0 \\
& \Longleftrightarrow Q_{n}(z)=0 .
\end{aligned}
$$

Note Note that $Q_{n}(z)=0$ is an equation of real coefficients. It, therefore, has complex conjugate roots.

From the above discussion, we can obtain the conclusion as follows:

Corollary 3.2 If $\alpha \pm \mathbf{i} \beta, \beta>0$, is a solution of $Q_{n}(z)=0$, then $\alpha+\beta \underline{\omega}$ is a spherical zero of $Q_{n}(x)$.

Proposition 3.2 Given a polynomial $Q_{n}(x)$ of real coefficients. Then there exists a one-to-one correspondence between its real isolated zeroes and the real roots of $Q_{n}(z)$, as well as a one-to-one correspondence between the spherical zeroes of $Q_{n}(x)$ and the pairs of complex conjugate zeroes of $Q_{n}(z)$.

Using Proposition 3.2, we have

Theorem 3.1 Given a polynomial $Q_{n}(x)$ with real coefficients, it has at least one zero. The zeroes are either isolated real ones or spherical zeroes. A pair of complex conjugate roots of $Q_{n}(x)$ with multiplicity $k, 2 k \leq n$, corresponds to a single spherical zero. The 
number of the isolated real zeroes together with the double number does not exceed the degree of the polynomial.

Examples 1 For $x^{2}-p x+q=0$, where $p, q \in \mathbf{R}$, considering $z^{2}-p z+q=0$, we have

$$
z_{1,2}=\frac{p \pm \sqrt{p^{2}-4 q}}{2}
$$

If $p^{2}-4 q \geq 0$, then $x_{1,2}=\frac{p \pm \sqrt{p^{2}-4 q}}{2}$ are zeroes of $x^{2}-p x+q=0$. If $p^{2}-4 q<0$, then $x=\frac{p}{2}+\frac{\sqrt{4 q-p^{2}}}{2} \underline{\omega}$ is a spherical zero of $x^{2}-p x+q=0$.

From Example 1, we obtain that

Corollary 3.3 There is a one-to-one correspondence between the spherical zero $x=\alpha+r \underline{\omega}$ and the equations of real coefficients $\left[x^{2}-2 \alpha x+\left(r^{2}+\alpha^{2}\right)\right]^{k}=0, k=1,2, \cdots$.

Examples 2 For $x^{3}+2 x^{2}+x+2=0$, considering $z^{3}+2 z^{2}+z+2=0$, we have

$$
z_{1,2}= \pm \mathbf{i}, z_{3}=-2
$$

Then zeroes of $x^{3}+2 x^{2}+x+2=0$ are $x_{1}=\underline{\omega}$ and $x_{2}=-2$.

Example 3 For $x^{4}+x^{2}+1=0$, considering $z^{4}+z^{2}+1=0$, we have

$$
\begin{gathered}
z_{1,2}=\frac{1}{2}(1 \pm \sqrt{3} \mathbf{i}) \\
z_{3,4}=-\frac{1}{2}(1 \pm \sqrt{3} \mathbf{i}) .
\end{gathered}
$$

The zeroes of $x^{4}+x^{2}+1=0$ are $x_{1,2}= \pm \frac{1}{2}(1+\sqrt{3} \underline{\omega})$.

Before proving Theorem 2.1, we need a Lemma.

Lemma 3.1 If $\alpha_{1}+r_{1} \underline{\omega}, \alpha_{2}+r_{2} \underline{\omega}, \cdots, \alpha_{p}+r_{p} \underline{\omega},\left(1 \leq p \leq\left[\frac{n}{2}\right]\right)$ are different spherical zeroes of $R_{n}$ with multiplicity $k_{1}, \cdots, k_{p}$, then the polynomial can be written as

$$
R_{n}(x)=R_{n-2 k_{p}}(x)\left[x^{2}-2 \alpha_{1} x+\left(r_{1}^{2}+\alpha_{1}^{2}\right)\right]^{k_{1}} \cdots\left[x^{2}-2 \alpha_{p} x+\left(r_{p}^{2}+\alpha_{p}^{2}\right)\right]^{k_{p}} .
$$

Proof For $R_{n}(x)=A(x) x+B(x)$. If $w_{1}=\alpha_{1}+r_{1} \underline{\omega}$ is a spherical zero of $R_{n}(x)$, then $\overline{w_{1}}$ is also a zero of it. As the proof of Proposition 2.1, we have $A\left(w_{1}\right)=A\left(\overline{w_{1}}\right)=0$ and $B\left(w_{1}\right)=B\left(\overline{w_{1}}\right)=0$. Using the properties of $A(x)$ and $B(x)$, we obtain that $A\left(\alpha_{1} \pm \mathbf{i} r_{1}\right)=$ 0 and $B\left(\alpha_{1} \pm \mathbf{i} r_{1}\right)=0$. Therefore, $R_{n}\left(\alpha_{1} \pm \mathbf{i} r_{1}\right)=0$. We have

$$
R_{n}(z)=R_{n-2}(z)\left[z-\left(\alpha_{1}+\mathbf{i} r_{1}\right)\right]\left[z-\left(\alpha_{1}-\mathbf{i} r_{1}\right)\right]=R_{n-2}(z)\left[z^{2}-2 \alpha_{1} z+\left(r_{1}^{2}+\alpha_{1}^{2}\right)\right] .
$$

Hence $R_{n}(x)=R_{n-2}(x)\left[x^{2}-2 \alpha_{1} x+\left(r_{1}^{2}+\alpha_{1}^{2}\right)\right]$. Using a finite number of iterations of this procedure leads to the conclusion. This completes the proof. 
Proof of Theorem 2.1 Let $\alpha_{1}+r_{1} \underline{\omega}, \alpha_{2}+r_{2} \underline{\omega}, \cdots, \alpha_{p}+r_{p} \underline{\omega},\left(1 \leq p \leq\left[\frac{n}{2}\right]\right)$ be different spherical zeroes and $w_{2 p+1}, \cdots, w_{n+1}$ be different isolated zeroes of $R_{n}$. From Lemma 3.1, we know that the polynomial can be written as

$$
R_{n}(x)=R_{n-2 k_{p}}(x)\left[x^{2}-2 \alpha_{1} x+\left(r_{1}^{2}+\alpha_{1}^{2}\right)\right]^{k_{1}} \cdots\left[x^{2}-2 \alpha_{p} x+\left(r_{p}^{2}+\alpha_{p}^{2}\right)\right]^{k_{p}}
$$

for some integers $k_{1}, k_{2}, \ldots, k_{p}$, and thus the degree of $R_{n-2 k_{p}}(x)$ is less than $n-2 p+1$. If $R_{n}\left(w_{i}\right)=0,2 p+1 \leq i \leq n+1$, note that $w_{i}^{2}-2 \alpha_{j} w_{i}+\left(r_{j}^{2}+\alpha_{j}^{2}\right), 2 p+1 \leq i \leq n+1,1 \leq j \leq p$ have inverse vectors, then $R_{n-2 k_{p}}\left(w_{i}\right)=0$. That means equation $R_{n-2 k_{p}}(x)=0$ has $n-2 p+1$ isolated zeroes, which is a contradiction to Proposition 2.3. This completes the proof.

\section{References}

[1] Brackx F, Delanghe R, Sommen F. Clifford Analysis. (Research Notes in Mathematics, Vol. 76), Boston, London, Melbourne: Pitman Advanced Publishing Company, 1982 .

[2] Delanghe R, Somman F, Soucek V. Clifford Algebra and Spinor-Valued Functions. Dorderecht, Boston, London: Kluwer Academic Publishers, 1992.

[3] Niven I. Equations in quaternions. Amer. Math, Monthly, 1941, 48: 654-661

[4] Niven I. The roots of quaternions. Amer. Math, Monthly, 1942, 49: 386-388

[5] Eilenberg S, Niven I. The fundamental theorem of algebra for quaternions. Bull. Amer. Math. Soc, 1994, 50: 246-248

[6] Pogorui A, Shapiro M. On the structure of the set of zeroes of quaternionic polynomials. Complex Variables, 2004, 49(6): 379-389

[7] Datta B, Nag S. Zero-sets of quaternionic and octonionic analytic functions with central coefficients. Bulletin of the London Mathematical Society, 1987, 19(4): 329-336

[8] Pogorui A A, Rodriguez-Dagnino R M. On the set of zeroes of bicomplex polynimials. Complex Variables and Elliptic Equations, 2006, 51(7): 725-730

[9] Sommen F. Some connections between Clifford analysis and complex analysis. Complex Variables Theory Appl, 1982, 1(1): 97-118

[10] McIntosh A. Clifford algebras, Fourier theory, singular integrals, and harmonic functions on Lipschitz domains. In: Clifford Algebras in Analysis and Related Topics (Fayetteville, Ark, 1993), Boca Raton, Florida: Stud. Adv. Math. CRC press, 1996. 33-87 